\title{
Apoptosis in a Canine Model of Acute Chagasic Myocarditis
}

\author{
Jun Zhang, Zilton A. Andrade, Zu-Xi Yu, Sonia G. Andrade, \\ Kazuyo Takeda, Mosés Sadirgursky and Victor J. Ferrans \\ Pathology Section, National Heart, Lung \& Blood Institute, National Institutes of Health, Bethesda, \\ MD 20892, USA and Division of Applied Pharmacology Research, Food and Drug Administration, \\ Laurel, MD 20708, USA and Ministerio da Saude, Fundaçao Oswaldo Cruz, Centro de Pesquisas \\ Gonçalo Muñiz, Rúa Valdemar Falçao, 121-Brotas, Salvador, BA 41.945, Brasil
}

(Received 23 September 1998, accepted in revised form 30 November 1998)

\begin{abstract}
J. Zhang, Z. A. Andrade, Z.-X. Yu, S. G. Andrade, K. Takeda, M. Sadirgursky and V. J. Ferrans. Apoptosis in a Canine Model of Acute Chagasic Myocarditis. Journal of Molecular and Cellular Cardiology (1999) 31, 581-596. Histologic, ultrastructural and nick end labeling studies were made to evalute the occurrence of apoptosis in the hearts of dogs with acute myocarditis due to experimental infection with T. cruzi. The best results for the detection of apoptosis by nick end labeling were obtained by a method combining the use of terminal deoxynucleotidyl transferase, $\mathrm{CoCl}_{2}$ and fluorescein-conjugated deoxyuridine triphosphate, followed by counterstaining of DNA with 4'6-diamidino-2-phenylindole (DAPI) and examination by laser scanning confocal fluorescence microscopy. Apoptosis was found in: (1) cardiac myocytes; (2) endothelial cells of capillaries and venules; (3) immune effector cells, including macrophages, interstitial dendritic cells (antigen-presenting cells) and granular and agranular lymphocytes, and (4) intra- and extracellular forms of T. cruzi. The apoptosis in myocytes and endothelial cells affected cells that were not infected by T. cruzi and was probably caused by the release of toxic mediators of inflammation. The apoptosis of immune effector cells could be related either to the subsidence of inflammation or to modulation (and even failure) of the immune response. The finding of apoptosis in T. cruzi confirms the results of other studies showing that this phenomenon occurs during the differentiation of trypomastigotes in vitro. Thus, apoptosis constitutes an important and multifactorial event in the pathogenesis of acute Chagasic myocarditis.

(C) 1999 Academic Press
\end{abstract}

KeY WoRDS: Trypanosoma cruzi; apoptosis; acute myocarditis; dog; nick end labeling; electron microscopy.

\section{Introduction}

Chagas' disease (American trypanosomiasis) is caused by the protozoan parasite Trypanosoma cruzi (T. cruzi). The most prominent manifestation of Chagas disease is related to the involvement of the cardiovascular system, and is initially characterized by acute myocarditis. In this phase of the disease, trypomastigotes invade cardiac muscle cells, where they become amastigotes (aflagellate forms), which then multiply and differentiate into trypomastigotes. These changes induce distention and eventual rupture of the invaded myocytes, with the release of the parasites into the interstitium
(Andrade et al., 1994). It has been suggested that the number of amastigotes that multiply in a cell is under genetic control, and that their differentiation depends on a programmed number of cell divisions (De Souza, 1984; Billaut-Mulot et al., 1996). Recent studies have shown that apoptosis occurs during the in vitro differentiation of epimastigotes into trypomastigotes of T. cruzi (Ameisen et al., 1995) and T. brucei rhodesience (Welburn et al., 1996). However, no information is available on the occurrence of apoptosis during the in vivo differentiation and multiplication of T. cruzi in myocardium.

In a canine model of acute Chagasic myocarditis,

Please address correspondence to: Victor J. Ferrans, MD, PhD, Chief, Pathology Section, National Heart, Lung and Blood Institute, Bld. 10/2N240, National Institutes of Health, 10 Center Dr. MSC-1518, Bethesda, MD 20892-1518, USA. 
we have observed that much of the myocyte damage occurs in cells that are not invaded by T. cruzi but are in close contact with mononuclear inflammatory cells (Andrade et al., 1994). These observations suggest that apoptosis induced by immune mechanisms can lead to the death of myocytes and other parenchymal cells of the heart in acute Chagasic myocarditis. In addition, increasing evidence suggests that apoptosis of T-lymphocytes plays a crucial role in modulating host immune responses and in balancing host-parasite interactions in Chagas' disease (Lopes and Dosreis, 1995; Lopes et al., 1995a, b; Dosreis et al., 1995). In a murine model of Chagas disease, apoptosis has been shown to occur in vitro in $\mathrm{CD}^{+}$lymphocytes derived from spleen, and in both $\mathrm{CD} 4^{+}$and $\mathrm{CD} 8^{+}$ lymphocytes in vivo (Lopes et al., 1995b). Thus, these studies suggest that apoptosis involving both the infecting organisms and various host cells is of importance in the pathogenesis of acute Chagasic myocarditis. To test this hypothesis, we have employed nick end labeling (for the in situ detection of DNA fragmentation) and ultrastructural techniques to identify apoptosis in cardiac myocytes, other parenchymal cells and immune effector cells, as well as in T. cruzi organisms, in a canine model of acute Chagasic myocarditis.

\section{Materials and Methods}

\section{Experimental animals}

The clinical, histological and electron microscopic features of the canine model used in this study have been described previously (Andrade et al., 1994). Briefly, six mongrel dogs weighing from 1000-1900 g were inoculated intraperitoneally with mouse blood containing T. cruzi trypomastigotes (the inoculum totalled $4 \times 10^{5}$ blood forms) of the 12 SF strain and sacrificed 18 days later. Two normal, non-infected dogs served as controls. At necropsy, multiple samples of heart were fixed with phosphate-buffered $10 \%$ formalin and embedded in paraffin for histologic and nick end labeling studies. For transmission electron microscopic study, other tissue samples were fixed with glutaraldehyde and processed as described previously (Andrade et al., 1994).

\section{Nick end labeling techniques}

Four different techniques were employed for nick end labeling. These techniques share the use of a pre-incubation step with proteinase $\mathrm{K}$ and incubation with a terminal deoxynucleotidyl transferase (TDT), but differ in the nature of the chromogen coupled to the deoxynucleotide substrate and in the divalent cation used to catalyze the reaction. A detailed comparison of the features of these four methods is presented in Table 1 . Commercially available kits were employed to carry out these methods, the manufacturer's instructions were followed, except where indicated.

The first of these methods, ApopTag peroxidase kit (Oncor, Gaithersburg, MD, USA) employs an incubation mixture that contains TDT, digoxigeninlabeled deoxynucleotides and $\mathrm{CoCl}_{2}$, followed by incubation with peroxidase-labeled anti-digoxigenin antibody and color development with diaminobenzidine tetrahydrochloride (DAB). This method requires inactivation of endogenous peroxidase activity by $\mathrm{H}_{2} \mathrm{O}_{2}$. Details of this method, as currently modified for use in our laboratory have been described (Zhang et al., 1996).

The second method (ApopTag fluorescent kit, Oncor) is similar to the first method, except for the use of anti-digoxigenin antibody conjugated to fluorescein isothiocyanate (FITC). This method was used in conjunction with examination of the preparations by confocal microscopy.

The third method (TACS 2 peroxidase kit, Travigen, Gaithersburg, MD, USA) also requires preincubation with $\mathrm{H}_{2} \mathrm{O}_{2}$ but differs from the methods described above in that it employs biotinylated deoxynucleotides, peroxidase-labeled streptavidin and a proprietary blue chromogenic substrate to reveal the sites of peroxidase reactivity. In addition, it uses $\mathrm{MnCl}_{2}$ instead of $\mathrm{CoCl}_{2}$ in the reaction mixture. It should be noted that the blue color resulting from this reaction is not permanent and shows substantial fading within a few days after color development.

The fourth method (ApopTag direct fluorescein kit, Oncor) features the use of deoxynucleotides labeled directly with FITC, which obviates the need for a secondary chromogenic reaction. This method uses $\mathrm{CoCl}_{2}$ in the incubation mixture.

As a positive control for nick end labeling, the sections were treated with proteinase $\mathrm{K}$, and then incubated with 250-500 $\mu \mathrm{g} / \mathrm{ml}$ DNase I (Sigma Chemical Co., St Louis, MO, USA) for $30 \mathrm{~min}$ at room temperature to induce partial cleavage of DNA (Gavrieli et al., 1992). For a negative immunohistochemical control, the sections were pretreated in the usual manner and then incubated in a reaction mixture from which the TDT had been omitted. 
Table 1 Comparison of the features of four methods for nick end labeling

\begin{tabular}{|c|c|c|c|c|c|c|c|}
\hline \multirow[t]{2}{*}{ Method } & \multirow[t]{2}{*}{ Source } & \multicolumn{2}{|c|}{ Pre-treatment } & \multicolumn{2}{|c|}{ TDT incubation medium, $1 \mathrm{~h}$} & \multirow[t]{2}{*}{ Chromogen } & \multirow[t]{2}{*}{ Counter-stain } \\
\hline & & Proteinase $\mathrm{K}$ & $\mathrm{H}_{2} \mathrm{O}_{2}$ & DNT label & Divalent cation & & \\
\hline Peroxidase-DAB & Oncor & $20 \mu \mathrm{g} / \mathrm{ml}, 15 \mathrm{~min}$ & $2 \%, 5 \min$ & Digoxigenin & $\mathrm{CoCl}_{2}$ & $\begin{array}{l}\text { Peroxidase-anti-digoxigenin } \\
\text { antibody and DAB }\end{array}$ & Methyl green \\
\hline Indirect-FITC & Oncor & $20 \mu \mathrm{g} / \mathrm{ml}, 15 \mathrm{~min}$ & NA & Digoxigenin & $\mathrm{CoCl}_{2}$ & FITC-anti-digoxigenin antibody & DAPI \\
\hline Peroxidase-TBL & Trevigen & $20 \mu \mathrm{g} / \mathrm{ml}, 15 \mathrm{~min}$ & $2 \%, 5 \min$ & Biotin & $\mathrm{MnCl}_{2}$ & TBL (Trevigen blue label) & Eosin \\
\hline Direct FITC & Oncor & $20 \mu \mathrm{g} / \mathrm{ml}, 15 \mathrm{~min}$ & NA & FITC & $\mathrm{CoCl}_{2}$ & NA & DAPI \\
\hline
\end{tabular}

Abbreviations: $\mathrm{DAB}=$ diaminobenzadine tetrahydrochloride; $\mathrm{DAPI}=4$ ', 6-diamidino-2-phenylindole; $\mathrm{DNT}=$ deoxynuclutide; FITC = fluorescein isothiocynate; TBL = Trevigen blue label $($ proprietary formulation); TDT = terminal deoxynucleotidyl transferase. 

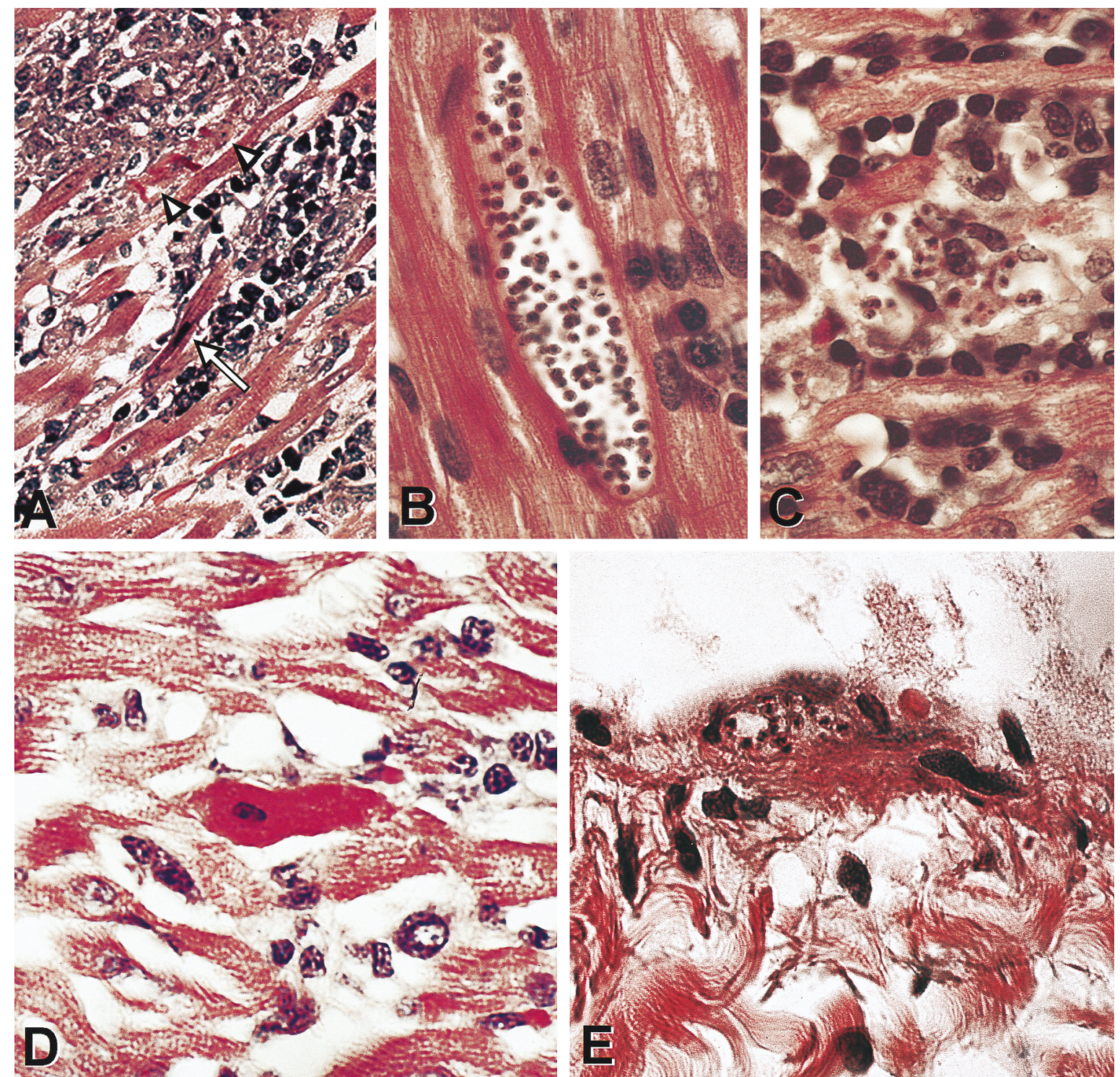

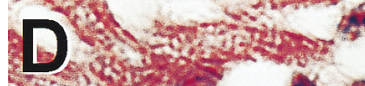
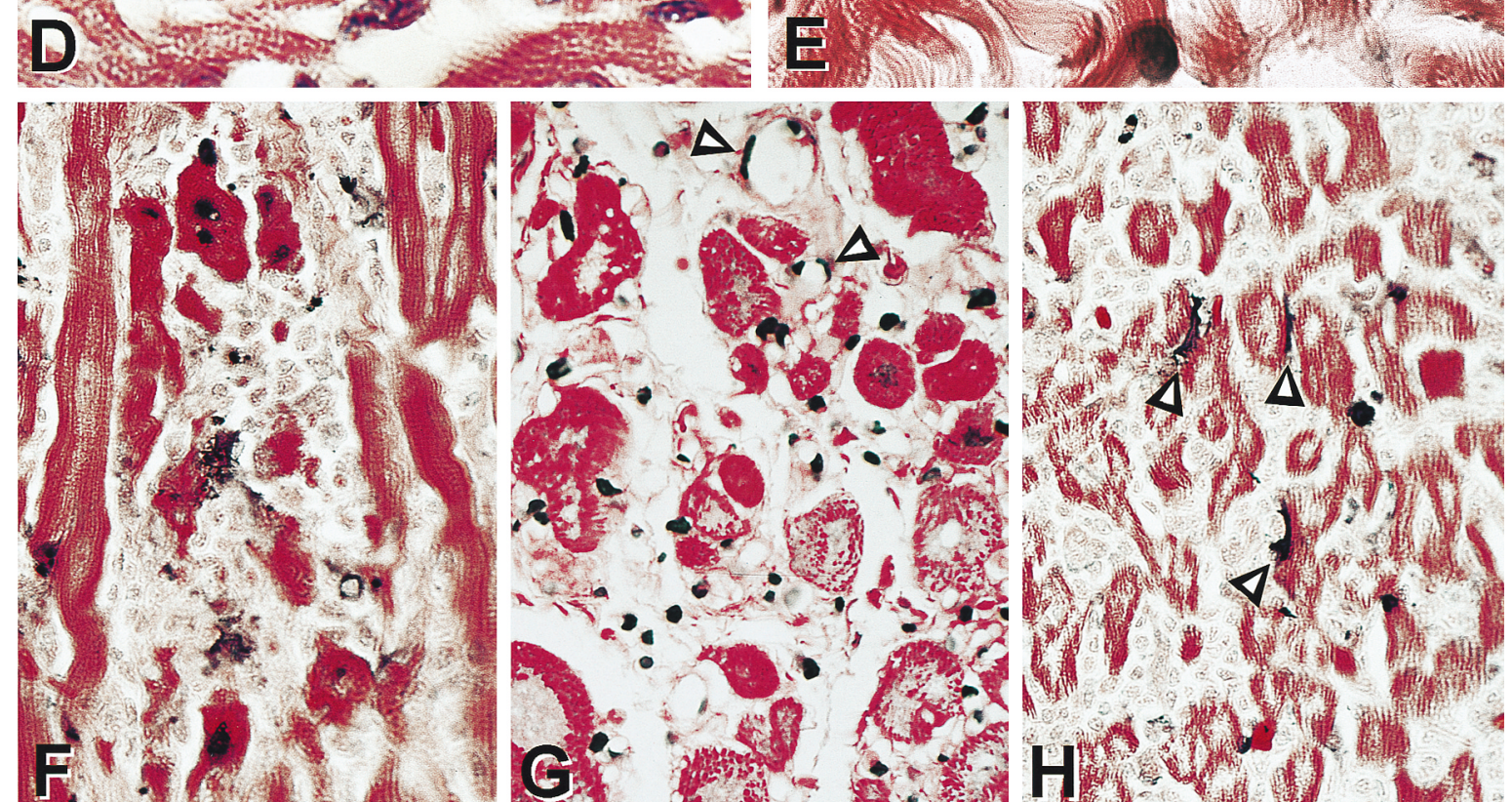


\section{Confocal microscopy}

Dual fluorescence microscopy methods were employed for the simultaneous detection of nick end labeling and nuclear staining. The sections were first stained by the nick end labeling methods 2 and 4 described above, and then counterstained with $0.01 \%$ 4'6-diamidino-2-phenylindole (DAPI) for $15 \mathrm{~min}$, then washed three times ( $5 \mathrm{~min}$ each) with PBS. The sections were then mounted with Vectashield medium (Vector Lab., Burlingame, CA, USA) and examined. In addition to blue fluorescent staining of the nuclei of all types of cells in myocardium, the use of this fluorochrome resulted in intense, distinctive staining of both the nucleus and the kinetoplast of intra- and extracellular forms of T. cruzi.

The sections processed by the dual staining methods described above were examined with a confocal laser scanning fluorescence microscope (Leica model TCS-4D DMIR-BE) equipped with argon and argon-krypton laser sources and $25 \mathrm{X}$ (planfluortar; N.A., 0.75), 40 X (planfluortar; N.A., 1.0) and $100 \mathrm{X}$ (planapochromat; N.A., 1.4) objectives. Green fluorescence scans (excitation, $488 \mathrm{~nm}$; emission, $525 \mathrm{~nm}$ ) for FITC labeling were performed separately from the blue scans (excitation $364 \mathrm{~nm}$; emission, $425 \mathrm{~nm}$ ) for DAPI. This was because the fluorescence emission spectrum of the latter reagent extends slightly into the range of the green emission detector, and this resulted in a spurious green signal when the two scans were performed simultaneously.

\section{Results}

\section{Histopathological observations}

The acute myocarditis caused by T. cruzi in dogs was characterized by the presence of amastigotes and differentiating trypomastigotes within the cytoplasm of cardiac myocytes and macrophages, as well as in the extracellular spaces, and by inflammatory infiltrates and myocardial degeneration and necrosis [Fig. 1(a)]. These lesions were very focal and varied considerably in size. In each of the animals, they were separated from adjacent lesions by areas of histologically normal myocardium. The myocytes invaded by T. cruzi contained clusters of up to 90 amastigotes [Fig. 1(b)], which occupied most of the cytoplasm, leaving only narrow, peripherally located zones containing compressed cytoplasmic organelles and a compact nucleus. Extracellular amastigotes [Fig. 1(c)] were more frequent in areas in which macrophages, lymphocytes and other inflammatory cells were present in large numbers, together with remnants of damaged or necrotic myocytes. Pronounced nuclear basophilia and other changes suggestive of nuclear condensation, similar to that seen in apoptosis in other cell types, were observed in some of the mononuclear inflammatory cells in edematous areas of the interstitium, as well as in some cardiac myocytes [Figs 1(a), (d)]. Intracellular amastigotes were occasionally found in endothelial cells of venules [Fig. $1(\mathrm{e})]$.

\section{Nick end labeling}

Each of the four methods employed for nick end labeling gave a positive reaction in the nuclei of some of the lymphocytes, macrophages, endothelial cells of capillaries and venules, interstitial dendritic cells and cardiac myocytes. The labeling of cardiac myocytes was much more clearly evident and distinct in the preparations stained either by the TACS blue method (method 3), or by the direct FITC labeling of the deoxynucleotides (method 4), than in those stained by the two techniques involving the use of digoxigenin-labeled deoxynucleotides

Figure 1 (opposite) Light micrographs showing cardiac histology [(a)-(e), hematoxylin and eosin stain] and nick end labeling for the detection of apoptosis [(f)-(h), method 3] in acute chagasic myocarditis. (a) Area of myocarditis, with severe inflammatory reaction and an apoptotic (arrow) and a necrotic (arrowheads) myocyte. The apoptotic cell has condensed nuclear chromatin and dark-staining cytoplasm. In contrast, the necrotic myocyte shows hypercontraction bands and partial loss of the nuclear chromatin; magnification, $\times 400$. (b) Numerous amastigotes are seen within the cytoplasm of a myocyte which has compressed cytoplasm and a compact nucleus. The amastigotes are characterized by a rounded nucleus and a bar-shaped kinetoplasts; magnification, $\times 1000$. (c) Extracellular amastigotes are present in the interstitium. A macrophage contains ten amastigotes in its cytoplasm; magnification, $\times 1000$. (d) A myocyte with darkly stained nucleus and deeply eosinophilic cytoplasm, suggestive of apoptosis; magnification, $\times 1000$. (e) Amastigotes are present within the cytoplasm of an endothelial cells of a venule; magnification, $\times 1000$. (f)-(h) Nick end labeling with the TACS blue (method 3, counterstained with eosin), shows a positive reaction (black colour) in the nuclei of: some myocytes situated in the periphery of an inflammatory focus (f); capillary endothelial cells (arrowheads) and lymphocytes $(\mathrm{g})$, and three interstitial dendritic cells [(h), arrowheads], which are identified by their shape and location; magnification, (f) and $(\mathrm{g}) \times 400$, (h) $\times 630$. 
(methods 1 and 2). More importantly, the use of methods 3 and 4 resulted in nick end labeling of some of the amastigotes of T. cruzi. The staining of these parasites by the other methods was inadequate. Negative control procedures (omission of the TDT) for each of the four methods gave negative rsults.

Nick end labeling of cardiac myocytes was almost exclusively present in cell that were not the sites of parasitic invasion. Such cells often were in actual contact with, or in close proximity to, mononuclear inflammatory cells. Myocytes that gave a positive reaction for nick end labeling most often were deeply eosinophilic and showed evidence of cytoplasmic condensation [Fig. 1(f)]. Nick end labeling was observed only very rarely in myocytes that contained clusters of amastigotes, although some of these organisms gave a positive reaction for nick end labeling. Cardiac myocytes present in noninflamed areas of myocardium did not show nick end labeling. Apoptotic bodies were not detected within cardiac myocytes. A few scattered apoptotic bodies were found in the cytoplasm of macrophages or were free in the interstitial spaces. Only few (less than 3\%) endothelial cells [Fig. 1(g)] showed nick end labeling, and such cells were present in areas of obvious inflammation. Some interstitial dendritic cells [Fig. 1(h)] showed nick end labeling, and they were identified on the basis of criteria previously described in detail (Zhang et al., 1993). This identification was confirmed by transmission electron microscopy (see below).

In preparations stained with DAPI, the globular structure of the nuclei of T. cruzi was clearly demonstrated. The kinetoplasts were discoid-shaped in some organisms; however, depending on the plane of sectioning, they often appeared as rods or bars [Fig. 2(a)]. Positive control procedures (DNA nicking induced by DNase I) produced positive reactions in all nuclei of tissue cells, and in both the nuclei and the kinetoplasts of T. cruzi [Fig. 2(b)]. In preparations counterstained with DAPI after nick end labeling with the direct FITC method, the green fluorescence of the nuclei of apoptotic myocytes was associated with nuclear condensation [Fig. 2(c)] and with nuclear fragmentation [Fig. 2(d)]. The green labeling of apoptotic amastigotes was clearly distinguishable from the blue staining of the DNA, in both the nuclei of myocytes and the nuclei and kinetoplasts of T. cruzi [Fig. 2(e)]. Amastigotes showing nick end labeling were found both in intracellular locations [Figs 2(e), (f)] and in the interstitium [Fig. 2(g)]. The patterns of nick end labeling in the amastigotes were variable. Some organisms showed a positive reaction in the nucleus and either a positive or a negative reaction in the kinetoplasts [Figs 2(e), (g)]. In other amastigotes, a positive reaction involved either the entire bar of the kinetoplasts or one or both of its lateral ends [Fig. 2(f)]. These various patterns of reactivity often coexisted within a cluster of intracellular amastigotes, and such clusters often contained a number of completely unreactive organisms. As many as ten apoptotic amastigotes could be found within the cytoplasm of a macrophage. Some apoptotic bodies had a bar-like shape, suggesting that they were phagocytosed kinetoplasts. In the interstitium, a large number of lymphocytes appeared to be undergoing apoptosis. Some of these lymphocytes were in close contact with extracellular amastigotes.

\section{Electron microscopic observations}

In addition to the general ultrastructural alterations that have been previously described in the canine model of acute Chagasic myocarditis (Andrade et al., 1994), electron microscopic studies disclosed various degrees of changes of nuclear and cytoplasmic condensation in some cardiac myocytes, endothelial cells, interstitial dendritic cells, macrophages and lymphocytes, as well as in amastigotes of T. cruzi.

In control dogs, electron microscopy study revealed that the chromatin in normal cardiac myocytes was finely dispersed, with a rim of heterochromatin marginated against the inner nuclear membrane [Fig. 3(a)]. The nuclei of myocytes which contained intracellular amastigotes showed greater margination of the chromatin. Much greater condensation of chromatin was observed in certain myocytes [Fig. 3(b)] that did not contain intracellular parasites, but were located in areas of inflammation, often in close proximity to inflammatory cells. These changes were often associated with cell shrinkage and increased electron density of the cytoplasmic organelles. Some myocytes showing these features appeared to have become fragmented. However, apoptopic bodies of the usual type, that is, containing highly dense clumps of nuclear material, were not identified in these remnants of myocytes. The coexistence of apoptotic and necrotic cardiac myocytes was generally observed in the periphery of inflammatory foci. In contrast to the high electron density of the nuclei and the cytoplasm of apoptotic myocytes, a low electron density of the cytoplasm (indicating myofibrillar loss, mitochondrial swelling, and dilatation of the sarcoplasmic reticulum) and nuclear swelling 

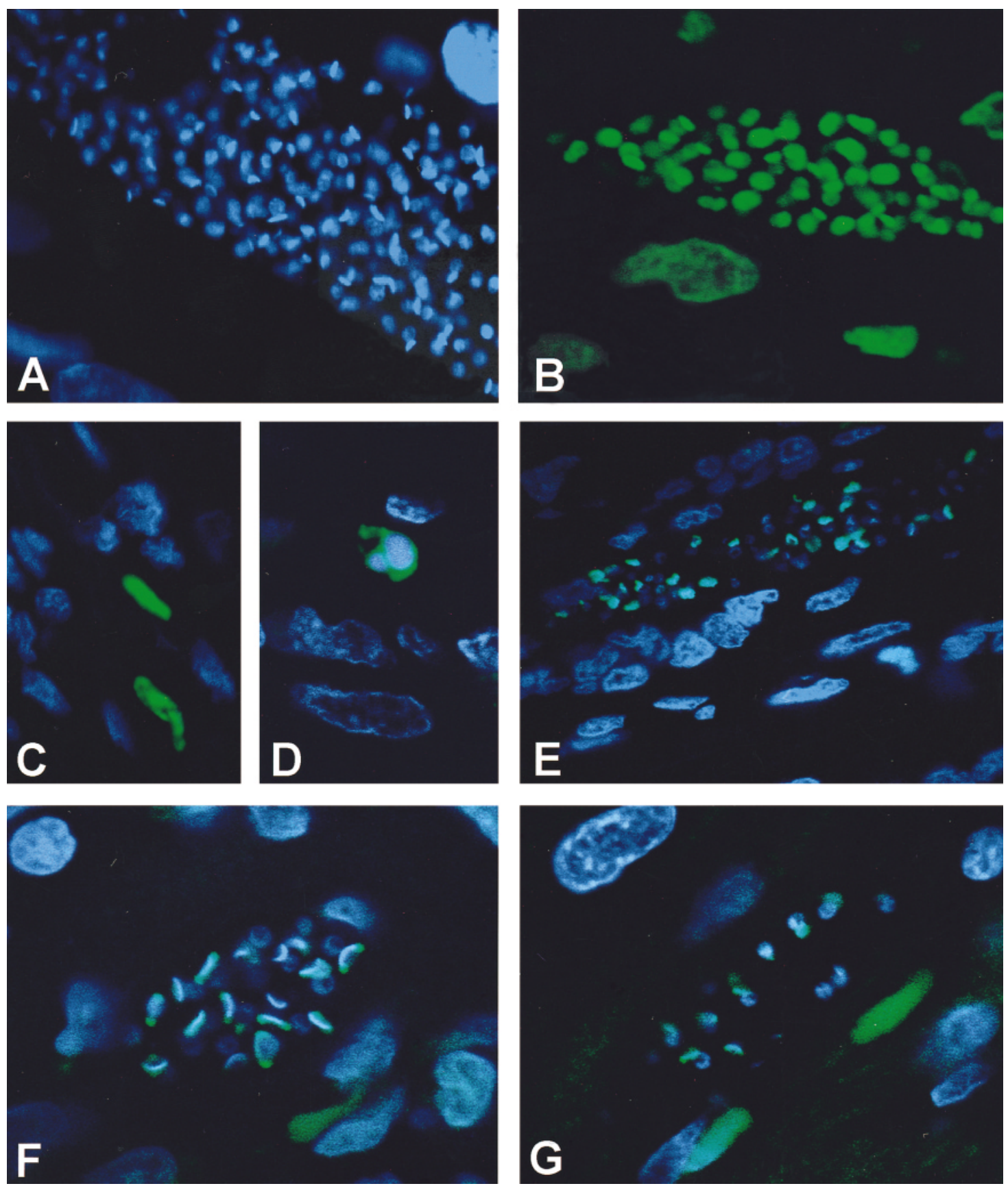

Figure 2 Confocal images of sections of myocardium showing: DAPI staining for DNA in blue and nick end labeling (direct FITC method, i.e. 4) for apoptosis in green. (a) Section stained only with DAPI shows intense fluorescence in the nuclei of various myocardial cells and in both the nuclei and the kinetoplasts of amastigotes; magnification, $\times 1000$. (b) Positive control section, pre-treated with DNase and stained only for nick end labeling, shows a reaction (green fluorescence) in nuclei of tissue cells and in both the nuclei and the kinetoplasts of T. cruzi. This reaction is similar to that demonstrated in (a) for DAPI; magnification, $\times 1200$. (c) and (d) Double staining shows green fluorescence (indicative of nick end labeling) in the condensed (c) or fragmented (d) nuclei of myocytes, and blue fluorescence (DAPI) in all other nuclei; magnification, (c) $\times 600$, (d) $\times 1000$. (e) Double staining shows combinations of green (nick end labeling) and blue (DAPI) in a cluster of amastigotes located within a cardiac myocyte. Some of these organisms are unreactive for nick end labeling and show only blue staining in both their nuclei and kinetoplasts. In other organisms, the nuclei show green labeling. The kinetoplasts in some of these organisms also show green labeling of the entire bar; magnification, $\times 600$. (f) Higher magnification view of cluster of amastigotes within a myocyte, showing green labeling limited to the lateral ends (green dots) of the kinetoplasts; magnification, $\times 1200$. $(\mathrm{g})$ Some extracellular organisms show a positive reaction in either the nuclei or the kinetoplasts, compare with 2(e). Unreactive nuclei are stained blue (DAPI); magnification, $\times 1000$. 

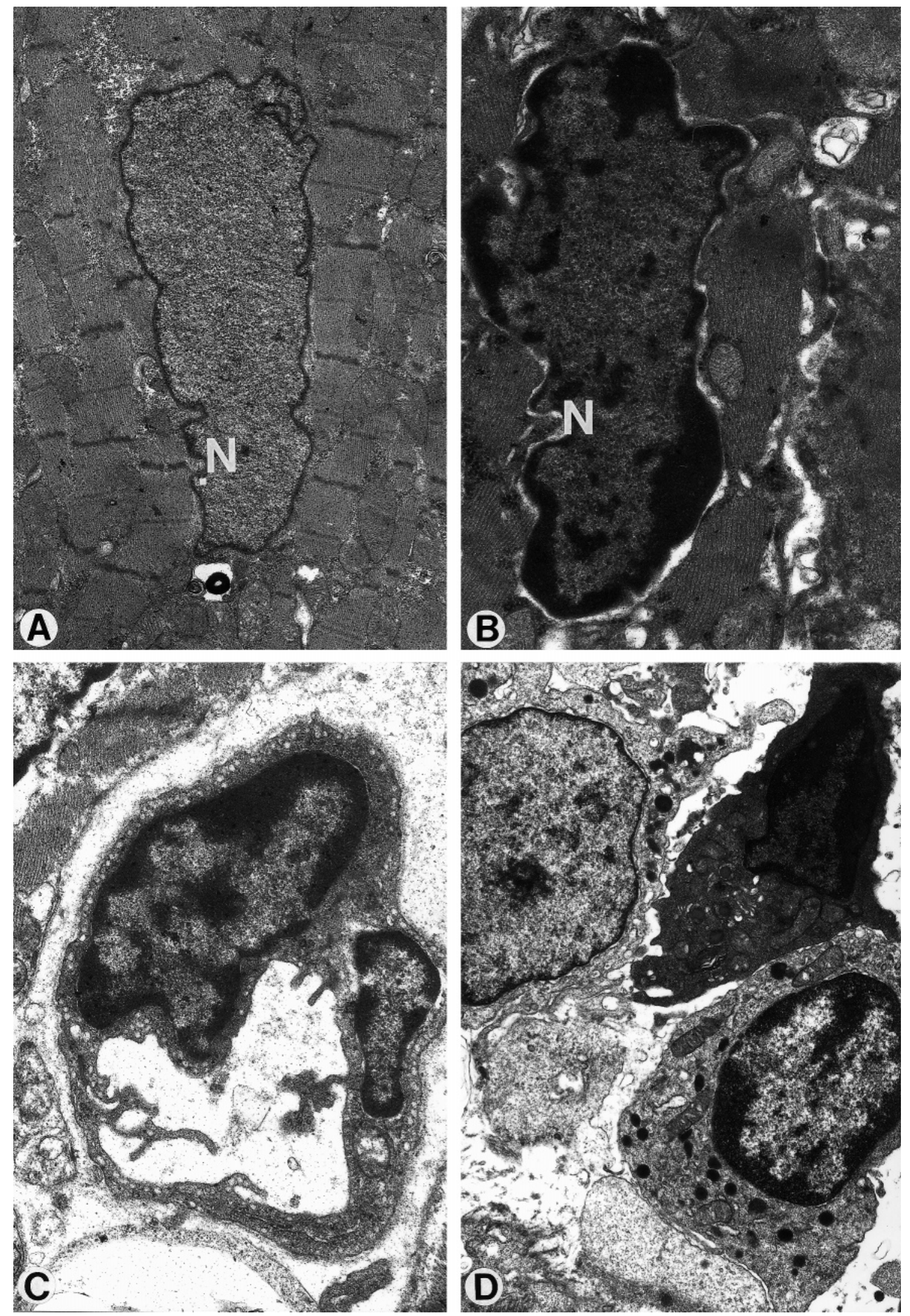
(indicating karyolysis) were often found in necrotic myocytes.

Condensation of the cytoplasm and the nuclear chromatin were also observed in a few endothelial cells of capillaries [Fig. 3(c)] and venules. The interstitial dendritic cells that appeared to be undergoing apoptosis had an irregular, elongated shape, several cytoplasmic processes, a few organelles and condensed nuclear chromatin. Macrophages had many lysosomes, phagolysosomes, slender cytoplasmic processes (filopodia), a large nucleus and well developed Golgi complexes. Amastigotes in various stages of disruption were present within some of the phagolysosomes. In some of the macrophages the nuclei were morphologically normal, in others they showed either lysis or pyknosis. Two ultrastructurally distinct types of lymphocytes were recognized: small agranular lymphocytes and large granular lymphocytes [Fig. 3(d)], which usually contained more than ten cytoplasmic granules. Apoptotic changes were observed more frequently in lymphocytes than in any other type of cell. Close contacts between antigen-presenting cells (macrophages and interstitial dendritic cells) and lymphocytes were frequently observed. Apoptotic bodies with small portions of fragmented nuclei and cytoplasm were found occasionally within the cytoplasm of lymphocytes (Fig. 4).

Individual amastigotes within the cytoplasm of cardiac myocytes were occasionally found to have intact nuclear membranes, moderately electron dense cytoplasm, and close contacts with myofilaments and mitochondria. These were presumed to be normal amastigotes in early stages of development in myocytes [Fig. 5(a)]. Some of the amastigotes present within cardiac myocytes also showed condensation of both the nucleus and the cytoplasm. In some of these amastigotes, the kinetoplast also appeared to be highly condensed. The shapes of the kinetoplasts varied from rod- or bar-like to slightly curved, discoid [Fig. 5(b)] or dumb-bell-like. Condensation of the mitochondrial DNA was evident in some kinetoplasts showing each of these variations in morphology. However, it often was clearly evident that the mitochondrial
DNA was more markedly condensed at the two ends of the kinetoplasts than in its central portion [Fig. 5(c)]. Furthermore, some amastigotes showed two small profiles of highly condensed intramitochondrial material that represented the two ends of the kinetoplasts (the central portion of which was not included in the plane of sectioning). While certain amastigotes located within a given myocyte showed changes of apoptosis [Fig. 5(d)], as described above, other amastigotes in the same cell exhibited changes that were indicative of necrosis [Fig. 5(d)]. These changes consisted of, mild nuclear swelling with rupture of the nuclear membranes, and decreased amounts and density of the chromatin; swelling and reduced electron density of the cytoplasm; variable numbers of cytoplasmic vacuoles, and rupture of the plasma membranes [Fig. 5(d)]. It is of interest to note that the kinetoplasts in these necrotic amastigotes had an increased electron density [Fig. 5(d)].

\section{Discussion}

The present study provides the first documentation of the occurrence of fragmentation of DNA, detected by nick end labeling, in the nuclei of some cardiac myocytes, endothelial cells of capillaries and venules, immune effector cells, and in both intra- and extracellular forms of T. cruzi in acute Chagasic myocarditis in dogs. Among the four different methods employed in the present study for the in situ detection of apoptosis, the best results were obtained with method 4, which uses fluoresceinconjugated dUTP and $\mathrm{CoCl}_{2}$ in the reaction mixture. This resulted in a bright staining of apoptotic nuclei, together with a dark background that was free of non-specific fluorescence. It has been suggested that the use of $\mathrm{CoCl}_{2}$ improves the specificity of the assay for staggered ends of the two cleaved strands of DNA. Although this method does not distinguish between double-strand cuts with one-base 3' overhangs and longer $3^{\prime}$ overhangs, it gives results that are similar to those obtained with the more specific method involving in situ ligation to 3' overhangs,

Figure 3 (opposite) Electron micrographs showing a normal myocyte (a), and apoptosis in a cardiac myocyte (b), an endothelial cell (c), and a large granular lymphocyte (d). (a) The nucleus (N) of a normal myocyte shows a peripheral, narrow rim of heterochromatins and abundant euchromatins evenly distributed within the nuclear matrix. Cytoplasmic structures appear normal; magnification, $\times 9000$. (b) Myocyte undergoing apoptosis shows sharply segregated, compact chromatin, convolved nuclear outline and condensed cytoplasm; N, nucleus; magnification, $\times 22$ 000. (c) An apoptotic capillary endothelial cell is characterized by segregation of the chromatin into sharply circumscribed areas subjacent to the inner nuclear membrane; magnification, $\times 15$ 000. (d) A large granular lymphocyte undergoing apoptosis shows cell shrinkage, compact chromatin and condensation of the cytoplasm. Compare with two other normal granular lymphocytes which have a large, round-shaped nucleus and electron-lucent cytoplasm; magnification, $\times 11000$. 


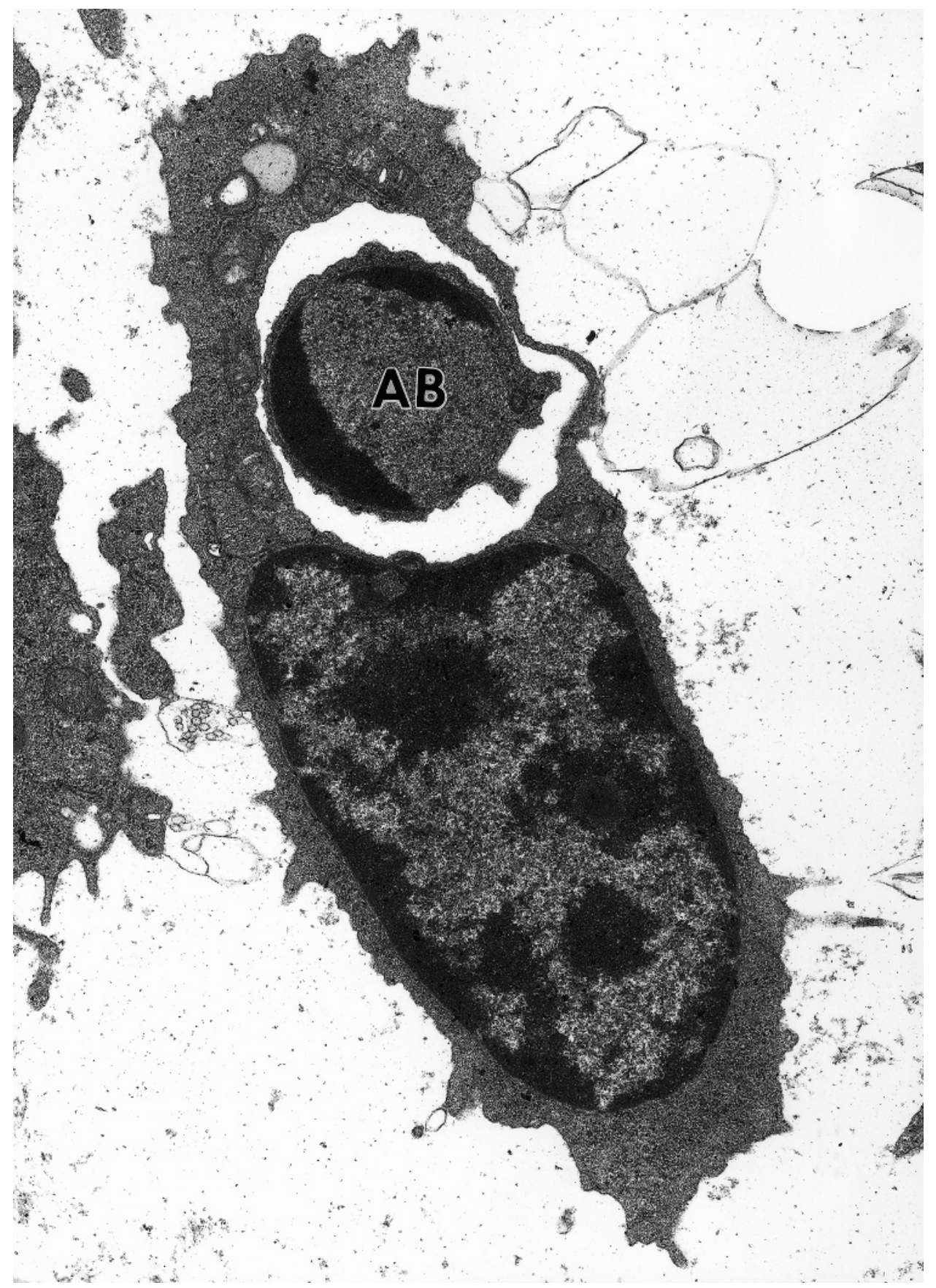

Figure 4 Electron micrograph showing an apoptotic body $(\mathrm{AB})$ seen in the cytoplasm of a lymphocyte. The apoptotic body is characterized by crescent-like condensation of nuclear chromatin, a narrow rim of cytoplasm, and a surrounding halo; magnification, $\times 25000$.

as demonstrated specifically with respect to cardiac myocytes (Anversa et al., 1998).

Quantitation of the extent to which apoptosis occurred in the canine model in the present study proved to be very difficult, because the area of myocarditis showed extreme variations in size, distribution and severity. Furthermore, our observations are to be considered as only preliminary, since we evaluated only one time point (considered to be that of maximal intensity) in the course of the acute myocarditis. The apoptosis observed in the present study was not evaluate by agarose gel electrophoresis for DNA laddering, because this method does not identify the diverse cellular sources of the cleaved DNA. Apoptosis is usually followed by little or no inflammation; 
however, the myocardial damage observed in the present study was associated with a considerable amount of inflammation. We atrribute this response to the coexistence of an inflammatory reaction directly elicited by the parasite, and by concomitant myocardial necrosis. Evidence of necrosis of myocytes was clearly observed in the present study, in the form of myofibrillar hypercontraction, karyolysis, hypereosinophilia, and cytoplasmic swelling. The degree to which these changes occurred was variable, as was their frequency. Although in many areas the incidence of these changes was comparable to that of the apoptotic changes observed by nick end labeling, it was not possible to determine to what extent they represented reversible or irreversible alterations. The necrosis may have resulted from toxic effects, alterations in the microcirculation, or cellular rupture due to distension by intracellular parasites.

\section{Apoptosis of cardiac myocytes}

Apoptosis of cardiac myocytes has been reported in ischemia-reperfusion in vivo (Gottlieb et al., 1994), hypoxia-reoxygenation in vitro (Tanaka et al., 1994), myocardial infarction (Kajstura et al., 1996; Sharov et al., 1996; Suzuki et al., 1996; Misao et al., 1996; Bardales et al., 1996; Cheng et al., 1996; Veinot et al., 1997), chronic myocarditis (Kawano et al., 1994), postnatal remodeling of the normal heart (Kajstura et al., 1995), cardiac hypertrophy (Bing, 1994), idiopathic dilated and ischemic cardiomyopathy (Narula et al., 1996), arrhythmogenic right ventricular dysplasia (James, 1994), rejection of transplanted hearts (Szabolcs et al., 1996), heart failure (Olivetti et al., 1997; Anversa et al., 1996), rapid ventricular pacing (Liu et al., 1995), mechanical stretch (Cheng et al., 1995), pressure overload due to aortic constriction (Teiger et al., 1996), aging in rats (Kajstura et al., 1996) and in spontaneously hypertensive rats and mice (Hamet et al., 1995). Thus, apoptosis has gained recognition as an important cause of myocyte death in a variety of pathological circumstances (Anversa et al., 1998). However, the occurrence of apoptosis in cardiac myocytes in Chagasic myocarditis has not been previously investigated by the nick end labeling technique. In our experience, this method was more sensitive and specific than electron microscopic study for the detection of apoptosis, because alterations in the ultrastructure of nuclei of cardiac myocytes proved extremely difficult to interpret.

In the present study, we observed the morphological and histochemical changes of apoptosis in cardiac myocytes, but did not find myocytederived apoptotic bodies. The presence of myofibrils may hinder the fragmentation of adult myocytes undergoing apoptosis. We have observed abundant apoptotic bodies in neonatal rat cardiac myocytes, in cultures in which apoptosis was induced by transfection with a recombinant adenovirus encoding for Id1, a nuclear protein that regulates differentiation and tissue-specific gene expression. The overexpression of Id 1 was associated with the increased production of reactive oxygen species by mitochondria, and by the development of apoptosis (Tanaka et al., 1998). In this context, apoptosis of cardiac myocytes also has been associated with the production of reactive oxygen species at the time of reperfusion following a period of temporary ischemia (Gottlieb et al., 1994), and in the rejection of transplanted hearts (Szaboles et al., 1996).

The apoptotic myocytes observed in the present study were not infected by T. cruzi, but frequently were in contact with macrophages or lymphocytes. These cells appear to be able to activate the pathway of programmed cell death in target cells and organisms (Helgason et al., 1993), probably by releasing various toxic mediators of inflammation. These findings provide further support to our earlier postulate (Andrade et al., 1994) that much of the damage to the myocardium during the acute phase of $T$. cruzi infection is mediated indirectly rather than by direct invasion of the cardiac myocytes by these parasites. Other studies have shown that immune reactions directed against various normal tissue components develop in Chagas' disease (Milei et al., 1993). It remains to be determined whether or not such phenomena can lead to apoptosis of cardiac myocytes in the chronic phase of this disease.

Our observations show that both apoptosis and necrosis of cardiac myocytes occur in acute Chagasic myocarditis. Apoptosis can be induced by many factors that are released from inflammatory cells, including TNF- $\alpha$ and other cytokines (Bowen and Bowen, 1990), nitric oxide (Szabolcs et al., 1996), reactive oxygen species (Buttke and Sandstrom, 1994), granzyme B (Heusel et al., 1994) and various growth factors (Bowen and Bowen, 1990). The specific causes of the apoptosis observed in various cell types were not determined in the present study.

\section{Apoptosis of endothelial cells}

Previous studies have demonstrated significant alterations, including aggregation of platelets and 

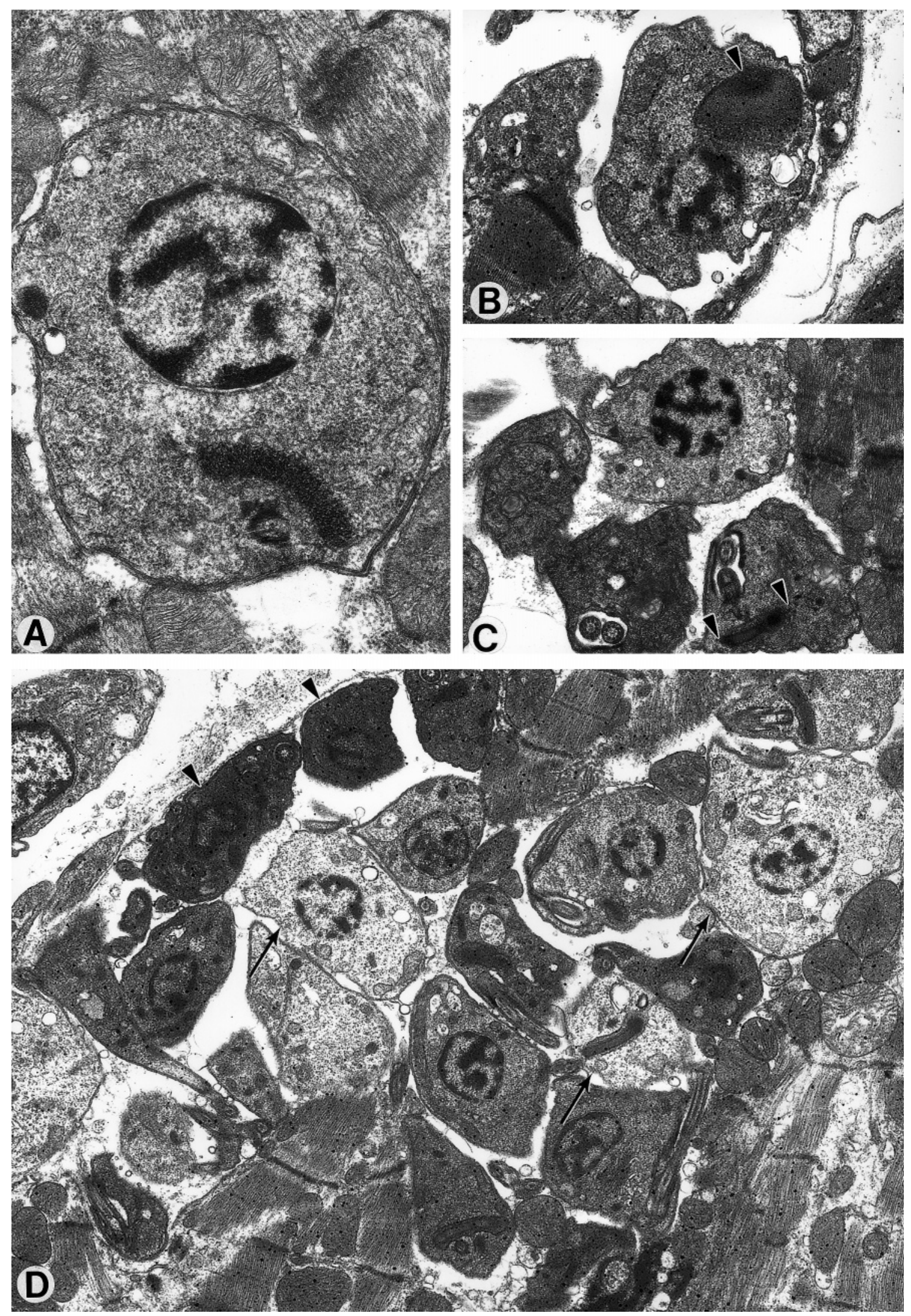
formation of microthrombi, in the myocardial microvasculature in acute Chagasic myocarditis (Andrade et al., 1994). The present study shows that apoptosis of endothelial cells occurs, but relatively infrequently, in cardiac capillaries and venules in acute Chagasic myocarditis. These changes were not associated with invasion of endothelial cells by T. cruzi (which was observed only rarely). It is likely that apoptosis of endothelial cells, like that of myocytes, is triggered by mediators of inflammation.

The detachment of endothelial cells from the underlying extracellular matrix rapidly leads to apoptosis, and the type of cell death that occurs under these circumstances has been termed anoikis. We have shown that this process is associated with an increase in reactive oxygen species in the affected endothelial cells, and that this increase is blocked by $\mathrm{N}$-acetylcysteine, diphenylene iodonium (a flavoprotein inhibitor) or by expression of a dominant negative form of the small GTPase rac1 (1998). These observations are of potential relevance to the apoptosis of endothelial cells in acute Chagasic myocarditis, since mediators of inflammation can cause the detachment of endothelial cells. This can lead to endothelial apoptosis and to microthrombosis associated with the direct exposure of the subendothelial connective tissue to blood.

\section{Apoptosis of immune effector cells}

We consider that the apoptosis observed in macrophages, lymphocytes and interstitial dendritic cells in the present study has a different functional significance than that found in cardiac myocytes and endothelial cells. Apoptosis of immune effector cells is critically important in the regulation and modulation of inflammatory responses (Golstein et al., 1991). It would appear that the apoptosis of lymphocytes in Chagas' disease has a very complex significance. Lopes et al. (1995b) observed apoptosis in vitro in splenic $\mathrm{CD} 4^{+} \mathrm{T}$ cells of mice infected with
T. cruzi. These authors believed that, after contact between the parasites and antigen-presenting cells, an effective immune response would require an appropriate interaction with parasite-specific $\mathrm{CD} 4^{+}$ $\mathrm{T}$ cells. Contact of $\mathrm{CD} 4^{+} \mathrm{T}$ cells with antigen at this critical stage results in their death by apoptosis (rather than in appropriate activation of the T cells), leading to failure of the immune response to destroy the parasites. This would facilitate the persistence of the parasites in tissues (Lopes et al., 1995b). In the present study, we observed a high incidence of apoptosis in both agranular and granular (i.e. CD8 ${ }^{+}$ or cytotoxic) lymphocytes. These findings are in agreement with those of Lopes et al. (1995b) who observed apoptosis of both $\mathrm{CD} 4^{+}$and $\mathrm{CD} 8^{+}$in vivo in experimental infection of mice with T. cruzi. The changes of apoptosis observed in lymphocytes in the present study can be interpreted as contributing to the subsidence of the acute inflammatory phase of the Chagasic myocarditis as well as to the elimination of $\mathrm{CD}^{+}$cells, as reported by Lopes and DosReis (1995) and Lopes et al. (1995b).

We have previously shown (Andrade et al., 1994) that in the dog model this acute phase is followed by the indeterminate phase, in which the hostparasite relationship is one of stable equilibrium, with only very few foci of continuing inflammation. It is of special interest to note the frequent occurrence of apoptosis in cells that had the morphological characteristics of interstitial dendritic cells, which are well known to serve as antigenpresenting and -processing cells. This apoptosis may be indicative of subsidence of inflammation, or may be a cause of failure of antigen presentation.

\section{Apoptosis of $T$. cruzi}

Recent studies have demonstrated that certain unicellular organisms can undergo apoptosis (Ameisen et al., 1995; Welburn et al., 1996), and that they can induce this phenomenon in target cells, as is the case in the killing of macrophages and neutrophils by entamoeba histolytica (Ragland et al.,

Figure 5 (opposite) Electron micrographs of normal, apoptotic and necrotic amastigotes of T. cruzi. (a) A single intracellular amastigote appears normal, showing intact nuclear membrane, normal distribution of nuclear chromatin, and a single mitochondrion with a centrally located kinetoplast; magnification, $\times 26000$. (b) One of the sides of a discoid-like kinetoplast shows a high electron density (arrowhead), but the nucleus and the cytoplasm of the parasites appear normal; magnification, $\times 12000$. (c) A high electron density is evident at the two ends (arrowheads) of a dumb-bell shaped kinetoplasts of an intracellular amastigote, the cytoplasm of this amastigote has a normal appearance; magnification, $\times 10000$. (d) A cluster of amastigotes is located within the cytoplasm of a cardiac myocyte. Some of these organisms show cell shrinkage and nuclear and cytoplasmic condensation indicative of apoptosis (arrowheads); by contrast, some other amastigotes show nuclear and cytoplasmic swelling, electron lucent cytoplasm and many cytoplasmic vesicles developing necrosis (arrows); magnification, $\times 9500$. 
1994). The occurrence of apoptosis during the course of the in vitro differentiation of epimastigotes into trypomastigotes has been demonstrated in $T$. cruzi (Ameisen et al., 1995) and in T. brucei rhodesiense (Welburn et al., 1996), by in situ nick end labeling and by agarose gel electrophoresis showing DNA ladders. The nuclear DNA in trypanosomes has a less complex organization than that of mammalian cells (Hecker et al., 1994). However, the organization of the mitochondrial DNA (known as kinetoplasts or $\mathrm{kDNA}$ ) in T. cruzi is much more complex than that in higher organisms.

T. cruzi contains a large amount of $\mathrm{kDNA}$, which is unusual in two respects, (1) it contains two different classes of circular DNA molecules, known as maxicircles and minicircles, and (2) in each kinetoplast these molecules are catenated to form a single, massive network (Stuart and Feagin, 1992). Each network contains 40-50 maxicircles (each, $33 \mathrm{~kb}$ ) and 5000-10 000 minicircles (each, 1.4 kb) (Stuart and Feagin, 1992). This is in contrast to human mitochondrial DNA, which is composed of a single class of non-catenated circular DNA molecules. The maxicircles of T. cruzi kDNA encode mitochondrial proteins, as is the case in mitochondrial DNA of higher eukaryotic cells. The minicircles function in RNA editing, which is extensive and serves to modulate mitochondrial gene expression (Stuart and Feagin, 1992). Replicated DNA is added at the periphery of the kDNA network and is subsequently redistributed. Alterations or loss of kDNA can occur rapidly under certain conditions (exposure to ethidium bromide or acridines) and may be lethal (Stuart and Feagin, 1992). However, biochemical changes in kDNA during apoptosis of trypanosomes have not been studied.

Welburn et al. (1996) reported that the apoptosis in epimastigotes of $T$. brucei rhodesiense did not involve kDNA, as shown by the lack of in situ nick end labeling in kinetoplasts. This finding is in agreement with the currently held concept that apoptosis does not involve mitochondrial DNA (Murgia et al., 1992; Tepper and Studzinski, 1992). Nevertheless, Ameisen et al. (1995) found what they considered to be "background staining" in the kinetoplasts of non-apoptotic epimastigotes stained for nick end labeling, and regarded this as due to the presence of numerous minicircles of DNA in the kinetoplasts. Their photographs show fluorescence localized to one or two dots in the cytoplasm of the epimastigotes, rather than diffuse staining of the whole bar of the kinetoplasts (Ameisen et al., 1995). However, we observed both partial and complete labeling of the kinetoplasts in some of the organisms that we studied. Furthermore, we detected various combinations of labeling of the nuclei and the kinetoplasts of T. cruzi. We attribute the nuclear staining to apoptosis; however, the significance of the partial or complete labeling of the kinetoplasts is uncertain. Fluorescence limited to portion(s) (usually, one or two dots corresponding to the ends of the bar) of the kinetoplasts but without coexistent nuclear fluorescence, is probably a normal finding (see below).

In electron micrographs of some amastigotes, we observed increased density of the two lateral ends of the bar forming the kinetoplasts. However, we do not know whether these alterations correspond to kDNA condensation. The nick end labeling that we observed in some kinetoplasts may have been related either to the replication of DNA in peripheral regions of the kDNA network, or strand breaks of unknown causes in the kDNA. The nuclear labeling would appear to be indicative of apoptosis, such as that detected in T. cruzi by the demonstration of DNA laddering (Ameisen et al., 1995).

Strong nick end labeling of the entire kinetoplasts were induced in control preparations treated with DNase I, thus confirming the susceptibility of kDNA to hydrolysis by this enzyme (the action of which is either very similar (Gottlieb et al., 1994) or identical (Anversa et al., 1998) to that involved in apoptosis. The studies of Welburn et al. (1996) and Ameisen et al. (1995) show that epimastigotes of both species of trypanosomes can undergo either necrosis or apoptosis, and that each of these processes can be induced and modulated independently by a number of factors. The present study demonstrates that both processes also occur in T. cruzi in vivo during the acute phase of Chagasic myocarditis in dogs. The possibility that the induction of apoptosis of $T$. cruzi may become useful in the therapy of Chagas' disease remains to be explored in detail. However, it has already been shown that apoptosis of $T$. cruzi can be induced by treatment with an antibiotic, geneticin (G418). This apoptosis is controlled by a T. cruzi elongation factor $1 \alpha$, which is present in the nucleus of the parasite (Billaut-Mulot et al., 1996).

In conclusion, the light microscopic, ultrastructural and nick end labeling studies presented in this report indicate that apoptosis occurs in a variety of cell types during the acute stage of the infection produced by $T$. cruzi in the hearts of dogs. Apoptosis was demonstrated in, (1) cardiac myocytes; (2) endothelial cells of capillaries and venules; (3) immune effector cells, that is, macrophages, granular and agranular lymphocytes, and interstitial dendritic cells, and (4) intracellular and extracellular forms of T. cruzi. Thus, apoptosis is an 
important mechanism of cell death and turnover in Chagasic myocarditis. The modulation of apoptosis may have therapeutic implications in Chagas' disease.

\section{Acknowledgements}

The authors would like to thank Mrs C. Jane Bell from the National Heart, Lung and Blood Institute, National Institutes of Health, Bethesda, MD for her expert editorial assistance.

\section{References}

Ameisen JC, Idziorek T, Billaut-Mulot O, Loyens M, Tissier JP, Potentier A, OuAissi A, 1995. Apoptosis in a unicellular eukaryote (Trypanosoma cruzi): implications for the evolutionary origin and role of programmed cell death in the control of cell proliferation, differentiation and survival. Cell Death Diff 2: 285-300.

Andrade ZA, Andrade SG, Correa R, SAdigursky M, FErRAns VJ, 1994. Myocardial changes in acute Trypanosoma cruzi infection. Ultrastructural evidence of immune damage and the role of microangiopathy. Am J Pathol 144: 1403-1411.

Anversa P, Kajstura J, Olivetti G, 1996. Myocyte death in heart failure. Curr Opin Cardiol 11: 245-251.

Anversa P, Annarosa L, Beltrami A, Guerra S, KAJSTuRA J, 1998. Myocyte death and growth in the failing heart. Lab Invest 78: 767-786.

Bardales RH, Hailey LS, Xie SS, Schaefer RF, Hsu SM, 1996. In situ apoptosis assay for the detection of early acute myocardial infarction. Am I Pathol 149: 821-829.

Billaut-Mulot O, Fernandez-Gomea R, Loyens M, QuAissi A, 1996. Trypanosoma cruzi elongation factor 1- $\alpha$ nuclear localization in parasites undergoing apoptosis. Gene 174: 19-26.

BING OH, 1994. Hypothesis. Apoptosis may be a mechanism for the transition to heart failure with chronic pressure overload [editorial] [see comments]. J Mol Cell Cardiol 26: 943-948.

Bowen ID, Bowen SM, 1990. Cell death and cell suicide in tumours. Anonymous (ed.) Programmed Cell Death in Tumours and Tissues. New York: Chapman and Hall, 84-86.

ButTKe TM, SANDSTrom PA, 1994. Oxidative stress as a mediator of apoptosis [see comments]. Immunol Today 15: $7-10$.

Cheng W, Li B, Kajstura J, Li P, Wolin MS, Sonnenblick EH, Hintze TH, Olivetti G, Anversa P, 1995. Stretchinduced programmed myocyte cell death. I Clin Invest 96: 2247-2259.

Cheng W, Kajstura J, Nitahara JA, Li B, Reiss K, Liu Y, Clark WA, Krajewsiki S, Reed JC, Olivetti G, Anversa P, 1996. Programmed myocyte cell death affects the viable myocardium after infarction in rats. Exp Cell Res 226: 316-327.

De Souza W, 1984. Cell biology of T. cruzi. Annu Rev Cytol 86: 197-283.
Dosreis GA, Fonseca ME, Lopes MF, 1995. Programmed Tcell death in experimental Chagas' disease. Parasitology Today 11: 390-394.

GAVRIELI Y, Sherman Y, Ben-SAsson SA, 1992. Identification of programmed cell death in situ via specific labeling of nuclear DNA fragmentation. J Cell Biol 119: 493-501.

Golstein P, Ojcius DM, Young JD, 1991. Cell death mechanisms and the immune system. Immunol Rev 121: 29-65.

GotTlieb RA, Burleson KO, Kloner RA, BABior BM, ENGLER RL, 1994. Reperfusion injury induces apoptosis in rabbit cardiomyocytes. J Clin Invest 94: 1621-1628.

Hamet P, Richard L, Dam TV, Teiger E, Orlov SN, Gaboury L, Gossard F, Tremblay J, 1995. Apoptosis in target organs of hypertension. Hypertension 26: 642-648.

Hecker H, Betschart B, Bender K, Burri M, Schlimme W, 1994. The chromatin of trypanosomes. Int J Parasitol 24: 809-819.

Helgason CD, Shi L, Greenberg AH, Shi Y, Bromley P, Cotter TG, Green DR, Bleackley RC, 1993. DNA fragmentation induced by cytotoxic T lymphocytes can result in target cell death. Exp Cell Res 206: 302-310.

Heusel JW, Wesselschmidt RL, Shresta S, Russell JH, LEY TJ, 1994. Cytotoxic lymphocytes require granzyme $B$ for the rapid induction of DNA fragmentation and apoptosis in allogeneic target cells. Cell 76: 977-987.

JAMES TN, 1994. Normal and abnormal consequences of apoptosis in the human heart. From postnatal morphogenesis to paroxysmal arrhythmias. Circulation 90: 556-573.

Kajstura J, Mansukhani M, Cheng W, Reiss K, Krajewski S, ReEd JC, Quaini F, Sonnenblick EH, Anversa P, 1995. Programmed cell death and expression of the protooncogene bcl-2 in myocytes during postnatal maturation of the heart. Exp Cell Res 219: 110-121.

Kajstura J, Cheng W, Sarangarajan R, Li P, Li B, Nitahara JA, Chapnick S, Reiss K, Olivetti G, ANVERSA P, 1996. Necrotic and apoptotic myocyte cell death in the aging heart of Fischer 344 rats. Am J Physiol 271: H1215-H1228.

Kajstura J, Cheng W, Reiss K, Clark WA, Sonnenblick EH, Krajewsiki S, Reed JC, Olivetti G, Anversa P, 1996. Apoptotic and necrotic myocyte cell deaths are independent contributing variables of infarct size in rats. Lab Invest 74: 86-107.

Kawano H, Okada R, Kawano Y, Sueyoshi N, Shirai T, 1994. Apoptosis in acute and chronic myocarditis. Jpn Heart I 35: 745-750.

Li AE, Ito H, Kim KS, Takeda K, Yu ZY, Ferrans VJ, FINKEL T, 1998. The role of reactive oxygen species in anoikis. J Biol Chem (Submitted).

Liu Y, Cigola E, Cheng W, Kajstura J, Olivetti G, Hintze TH, Anversa P, 1985. Myocyte nuclear mitotic division and programmed myocyte cell death characterize the cardiac myopathy induced by rapid ventricular pacing in dogs. Lab Invest 73: 771-787.

Lopes MF, DosReis GA, 1995. Apoptosis as a cause of Tcell unresponsiveness in experimental Chagas' disease. Brazilian J Med Biol Res 28: 913-918.

Lopes MF, Cunha JMT, Bezerra FL, Gonzalez MS, Gomes JEL, Silva JRLE, Garcia ES, Reis GAD, 1995a. Trypanosoma cruzi: both chemically induced and triatomine-derived metacyclic trypomastigotes cause the 
same immunological disturbances in the infected mammalian host. Exp Parasitol 80: 194-204.

Lopes MF, Da Veiga VF, Santos AR, Fonseca ME, Dosreis GA, 1995b. Activation-induced CD4 + T cell death by apoptosis in experimental Chagas' disease. J Immunol 154: 744-752.

Milei J, Sanchez J, Storino R, Yu ZX, Denduchis B, FERrANs VJ, 1993. Antibodies to laminin and immunohistochemical localization of laminin in chronic chagasic cardiomyopathy. A review. Mol Cell Biochem 129: 161-170.

Misao J, Hayakawa Y, Ohno M, Kato S, Fujiwara T, Fujiwara H, 1996. Expression of bcl-2 protein, an inhibitor of apoptosis, and Bax, an accelerator of apoptosis, in ventricular myocytes of human hearts with myocardial infarction. Circulation 94: 15061512 .

Murgia M, Pizzo P, Sandona D, Zanovello P, Rizzuto R, VIRGILIO F, 1992. Mitochondrial DNA is not fragmented during apoptosis. J Biol Chem 267: 10939-10941.

Nagata S, Golstein P, 1995. The Fas death factor. Science 267: 1449-1456.

Narula J, Haider N, Virmani R, Disalvo TG, Kolodgie FD, HajJar RJ, Schmidt U, Semigran MJ, Dec GW, KHAW BA, 1996. Apoptosis in myocytes in end-stage heart failure. N Engl J Med 335: 1182-1189.

Olivetti G, Abbi R, Quaini F, Kajstura J, Cheng W, Nitahara JA, Quaini E, Di Loreto C, Beltrami CA, Krajewsis S, Reed JC, Anversa P, 1997. Apoptosis in the failing human heart. N Engl J Med 336: 11311141.

Ragland BD, Ashley LS, Vaux DL, Petri WA Jr, 1994. Entamoeba histolytica. Target cells killed by Trophozoites undergo DNA fragmentation which is not blocked by Bcl-2. Exp Parasitol 79: 460-467.

Sharov VG, Sabbah HN, Shimoyama H, Goussev AV, LESCH M, GoldSTEIN S, 1996. Evidence of cardiocyte apoptosis in myocardium of dogs with chronic heart failure. Am J Pathol 148: 141-149.

Stuart K, Feagin JE, 1992. Mitochondrial DNA of kinetoplastids. Int Rev Cytol 141: 65-88.

SuZuki H, Wildhirt SM, Dudek RR, NARAyan KS, Bailey
AH, BING RJ, 1996. Induction of apoptosis in myocardial infarction and its possible relationship to nitric oxide synthase in macrophages. Tissue Cell 28: 89-97.

Szabolcs M, Michler RE, Yang X, Aji W, Roy D, Athan E, Sciacca RR, Minanov OP, Cannon PJ, 1996. Apoptosis of cardiac myocytes during cardiac allograft rejection. Relation to induction of nitric oxide synthase. Circulation 94: 1665-1673.

Tanaka M, Ito H, Adachi S, Акimoto H, Nishikawa T, Kasajima T, Marumo F, Hiroe M, 1994. Hypoxia induces apoptosis with enhanced expression of Fas antigen messenger RNA in cultured neonatal rat cardiomyocytes. Circ Res 75: 426-433.

Tanaka K, Pracy JB, Takeda K, Yu ZX, Ferrans VJ, Deshrande SS, Ozaki M, Hwang PM, Lowenstein CJ, IrANi K, FinKel T, 1998. Expression of Id1 results in apoptosis of cardiac myocytes through a redoxindependent mechanism. J Biol Chem 273: 2592225928.

Teigre E, Than VD, Richard L, Wisnewsky C, Tea BS, Gaboury L, Tremblay J, Schwartz K, Hamet P, 1996. Apoptosis in pressure overload-induced heart hypertrophy in the rat. J Clin Invest 97: 2891-2897.

TePPER CG, StudZinski GP, 1992. Teniposide induces nuclear but not mitochondrial DNA degradation. Cancer Res 52: 3384-3390.

Veinot JP, Gattinger DA, Eliss H, 1997. Early apoptosis in human myocardial infarcts. Hum Pathol 149: 821829.

Welburn SC, Dale C, Ellis D, Beecroft R, Pearson TW, 1996. Apoptosis in procyclic Trypanosoma brucei rhodesience in vitro. Cell Death Diff 2: 229-236.

Zhang J, Yu ZX, Fujita S, Yamaguchi ML, Ferrans VJ, 1993. Interstitial dendritic cells of the rat heart. Quantitative and ultrastructural changes in experimental myocardial infarction. Circulation 87: 909920.

Zhang J, Clark JR Jr, Herman EH, Ferrans VJ, 1996. Doxorubicin-induced apoptosis in spontaneously hypertensive rats: differential effects in heart, kidney and intestine, and inhibition by ICRF-187. J Mol Cell Cardiol 28: 1931-1943. 